\title{
Prevalence and Correlates of TB and HIV Co-infection Among People Living with HIVIAIDs at the DLHM Hospital, Calabar
}

\author{
Samson Olusegun Aturaka ${ }^{1}$, Abiodun Olaiya ${ }^{2}$, Olusola Omotola ${ }^{3}$, Wasiu Olalekan Adebimpe ${ }^{4}$, \\ Philip Imohi ${ }^{5}$, Affiang Daniel ${ }^{6}$, Enny Ogban ${ }^{7}$
}

${ }^{1}$ Department of Health System Strengthening and Laboratory Services, Fhi360 Cross River State Office, Calabar, Nigeria

${ }^{2}$ Department of Health System Strengthening and Logistics, Fhi360/AHNi Benue State Office, Calabar, Nigeria

${ }^{3}$ Department of Emergency Preparedness Services, Medecins Sans Frontieres French Section, Abuja, Nigeria

${ }^{4}$ Department of Community Medicine, Osun State University, Osogbo, Nigeria

${ }^{5}$ Department of Prevention, Care and Treatment, Fhi360 Cross River State Office, Calabar, Nigeria

${ }^{6}$ Department of Medical Laboratory Services, Lawrence Henshaw Memorial Hospital, Calabar, Nigeria

${ }^{7}$ Department of Medical Laboratory Services, General Hospital Calabar, Calabar, Nigeria

\section{Email address:}

segunhydd@yahoo.com (S. O. Aturaka),drabiodunolaiya@gmail.com (A. Olaiya),dasholi@outlook.com (O. Omotola), olalekanadebimpe@gmail.com (W. O. Adebimpe),phil_imohi@yahoo.com (P. Imohi)

\section{To cite this article:}

Samson Olusegun Aturaka, Olaiya Abiodun, Olusola Omotola, Wasiu Olalekan Adebimpe, Philip Imohi, Affiang Daniel, Enny Ogban. Prevalence and Correlates of TB and HIV Co-infection Among People Living with HIV/AIDs at the DLHM Hospital, Calabar. American Journal of Health Research. Vol. 5, No. 4, 2017, pp. 106-109. doi: 10.11648/j.ajhr.20170504.13

Received: January 27, 2017; Accepted: May 26, 2017; Published: July 12, 2017

\begin{abstract}
TB is the most common opportunity infection and cause of death among people living with HIV. With dwindling prevalence figures from several national studies and dwindling funding, CD4 monitoring makes management easy, accessible and affordable. Objectives to determine prevalence and correlates of TB and HIV Co-infection among People Living with HIV/AIDs at the DLHM Hospital, Calabar. The study is retrospective descriptive study of 274 TB-HIV co-infected clients form October, 2009 to June, 2011 was carried out by examining their hospital records. Research instruments were a designed checklist used to collect socio demographic data, information on HIV diagnosis and ARV use, CD4 count and TB-HIV coinfection. Data was analyzed using the excel software. The result shows age group 21-30 years and 31-40 years constitute the largest $(39.0 \%$ and $31.0 \%)$ of co-infected clients respectively. The percentage of infected females was $160 / 274=58.6 \%$ and the rest were male. Only $28(10.2 \%)$ had their CD4 count above 350 cells counts/ul. ConclusiCo-infections of TB and HIV are common. CD4 count is a reliable tool in monitoring progression. Every HIV positive client should be considered having the potential of contracting $\mathrm{TB}$, and this underscores the need for proper screening and monitoring.
\end{abstract}

Keywords: TB-HIV Co-infection, Prevalence, CD4 Cell Count

\section{Introduction}

Since the emergence of HIV, tuberculosis (TB) and HIV have been closely linked together. TB is the most common opportunity infection and cause of death among people living with HIV. [1] With a decrease in cell- mediated immunity, HIV changes the pathogenesis of $\mathrm{TB}$, thereby greatly increasing the risk of disease from TB in HIV-co-infected persons and progressing to more serious complications and forms of TB. In 2006, a total of 1.7 million people died of TB including 231,000 people with HIV. [2] [12] Nigeria have the fourth highest burden of tuberculosis (TB) in the world, with an annual incidence of 311 cases per 100,000 population and a mortality rate of 81 per 100,000 population in 2006. [4] [13] In Nigeria, about $21 \%$ of all TB patients are dually infected with TB and HIV. [3] [4]

In 2013 of the estimated 9 million people who developed $\mathrm{TB}$, an estimated 1.1 million (13\%) were HIV positive. There 
were also 360,000 deaths from HIV associated TB equivalent to $25 \%$ of all TB deaths, and around $25 \%$ of the estimated 1.5 million deaths from HIV/AIDS in the same year. [5] Factors contributing to the resurgence of tuberculosis in developing countries include co-infection with HIV; emergence of multiple resistant tuberculosis, inadequate treatment, poverty, malnutrition, overcrowding, armed conflict and increasing numbers of displaced persons.

In the course of HIV infection TB also occurs earlier than many other opportunistic infections. Also, in co-infected individuals the risk of death is twice that of HIV infected individuals without TB, even when CD4 cell count and antiretroviral therapy are taken into account. [8]

Many people are not aware of being infected with tubercle bacillus; the infection thus becomes reactivated by other infections such as the HIV when the body immune system is weakened. People living with HIV or immunosuppressed patients due to drugs seem more vulnerable to tuberculosis (TB) than HIV uninfected persons. HIV increases the progression of latent TB infection to active disease [9] and it is the cause of increase in the number of TB cases. [10] Brown [11] also reported highest rates of tuberculosis among infected people with HIV.

The uncertainty of moving from latent to active TB is estimated to be between 12 and 20 times greater in individual living with HIV than among those without HIV infection [7]. This also shows that they may become infectious and transmit TB to someone else, more quickly than would otherwise happen. Overall it is considered that the lifetime uncertainty for HIV negative individual of progressing from latent to active TB is about $5-10 \%$, whereas for HIV positive people this same figure is the annual risk. [8]

Without proper treatment, an estimated 90 percent of persons living with HIV die within months after contracting tuberculosis. [1] The initiation of highly active antiretroviral therapy (HAART) in persons co-infected with tuberculosis can cause an immune reconstitution inflammatory syndrome with a worsening, in some cases severe worsening, of tuberculosis infection and symptoms.

TB is often affected by the presence of other infections such as DM, housing factors, socio economic factors, poverty and malnutrition. These have made the prevalence of TB increased over the years despite the existence of DOTs programmes. TB-HIV co-infection has brought hardship into the existence of several communities and societies through morbidity, mortality, deformities, stigmatization and discrimination and poor health seeking behaviour. So studying co-infections would enhance the prospect of stakeholders achieving successes at disease control and prevention. The blood count of CD4 (cluster of differentiation 4) is a glycoprotein found on the surface of immune cells such as $\mathrm{T}$ helper cells, monocytes, macrophages, and dendritic cells is an important indicator of how well the immune system is improving, and it is being used as index of monitoring disease progression.

These epidemiologic relationships between TB and HIV, and the high prevalence of these diseases in sub-Saharan
Africa and indeed Nigeria make studying TB and HIV infected populations in this region of the world important. The malnutrition which is commonly present in patients with tuberculosis can add to the impaired antioxidant capacity in these patients. This study determines prevalence and correlates of TB and HIV Co-infection among People Living with HIV/AIDs at the DLHM Hospital, Calabar

\section{Methods}

The study area is the DLMIH hospital in Calabar in southwestern Nigeria. The hospital serves as referral centers to the numerous primary health care centers and mini cottage hospitals in the town. The HIV prevalence in Calabar is $7.1 \%$, a little above the Nigerian national prevalence rate put at $5.1 \%$. Between the periods October, 2009 to June, 2011, about 1960 clients were tested for HIV in the hospital out of which 475 were positive and enrolled into care. The hospital is being supported by an $\mathrm{NGO}$, with monitoring and supervisory roles being provided by the $\mathrm{FMoH}$.

This is a retrospective descriptive study of TB-HIV coinfection among HIV positive clients enrolled into care at the DLMIH hospital. Case notes of clients were reviewed to generate valuable research data. Eligible subject include a registered HIV positive client 18 years and above while those who were co-infected with HIV constitutes the direct study population.

All subjects who met the inclusion criteria were recruited into the study and their case notes examined for the relevant data under the cover of privacy and confidentiality. Research instrument consist of a designed checklist for collection of socio demographic data, information on HIV diagnosis and ARV use, CD4 count and TB-HIV co-infection

Approval to carry out this data was obtained from the chief medical director of the hospital and the site coordinator. A study limitation include few cases of missing data, thus subjects with complete data were eventually analyzed. Data was entered into the SPSS software version 17.0 after data cleaning and random checks. Frequency tables and charts were generated to showcase pattern of TB and HIV coinfections and some selected variables.

\section{Results}

Two hundred and seventy four patients co-infected with TB/HIV was recorded during this period from October, 2009 to June, 2011, this figure include 114 males (41.4\%) and 160 females $(58.6 \%) .13$ of the 274 patients were ages $<10$ representing $4.1 \%, 1$ patient of ages 11 to 20 representing $0.4 \%, 106$ patients of 21 to 30 representing $38.7 \%$. Others are 84 patients of ages 31 to 40 representing $30.1 \%$, 48 patients of ages 41 to 50 representing $17.5 \%$ and 22 patients that are above 50 years representing $8 \%$. Age group 21-30 years and $31-40$ years constitute the largest $(39.0 \%$ and $31.0 \%)$ of coinfected clients respectively (Figure 1). The percentage of infected females was $160 / 274=58.6 \%$ ) and the rest were male. (Figure 2). 
Table 1 shows the number of patients that are within the normal range of CD4 cell count $(500-1,600$ cell count/ul $)$ is 7 which represent $2.6 \%(\mathrm{P}=7 / 274=2.6 \%)$ of the total during the period under review. Also, the number of patients that there CD4 count are $>350$ cell count/ul is 28 representing $10.2 \%(\mathrm{P}=28 / 274)$ while those that are $<350$ represent $89.8 \%(\mathrm{P}=246 / 274)$.

\section{Number of Patients Co-infected with TB/HIV}

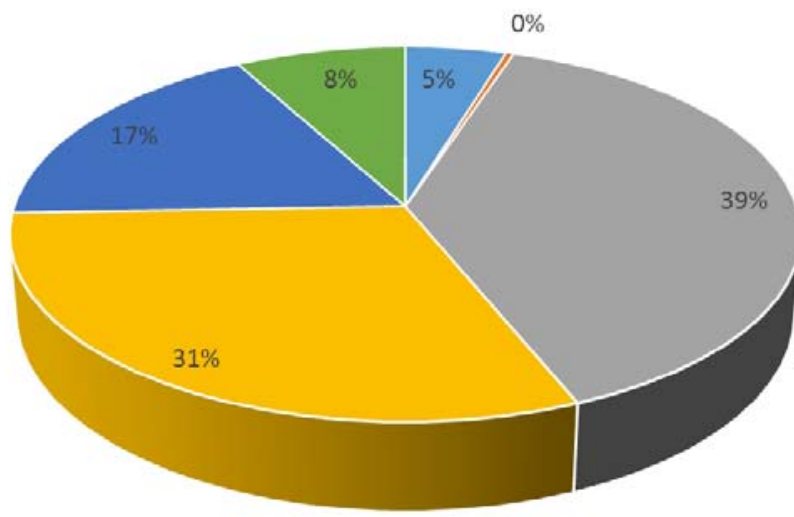

$=0-10$

- 20-Nov

$=21-30$

$=31-40$

- $41-50$

- 51 and Above

Figure 1. Age distribution of TB-HIV co-infected clients.

No of males or Females Co-infected with TB/HIV

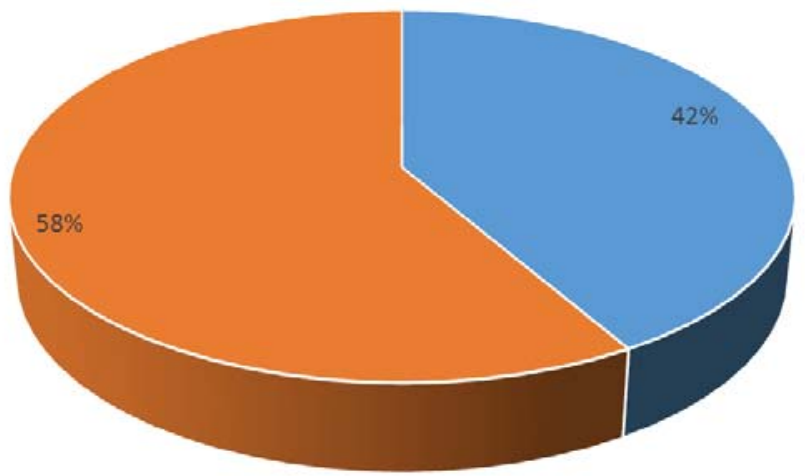

- No of Males

- No of Females

Figure 2. Sex distribution of TB-HIV co-infected clients.

Table 1. Correlates of TB/HIV co-infection.

\begin{tabular}{ll}
\hline & Co-infection \\
\hline CD4 count pattern & \\
$>350$ & $28(10.2 \%)$ \\
$<350$ & $246(89.8 \%)$ \\
Total & 274 \\
\hline
\end{tabular}

\section{Discussion}

The study revealed that females are more susceptible to TB/HIV co-infection than males by acquiring $58.6 \%$ of the total figure, the reason being that women bear a disproportionate burden of poverty, ill-health, malnutrition and disease worldwide. This is supported by a study 
conducted in 1996 by the World Bank, WHO and Harvard University that TB as a leading cause of "healthy years lost" among women of reproductive age. This is also true that women in this age group are at greater risk of becoming infected with HIV; hence the co-infection of these two deadly diseases had more impact on females than male.

The study also revealed that only $2.6 \%$ of the patients have their CD4 cell count within the normal range $<10.2 \%$ are not eligible for HAAT according to the WHO treatment guideline for HIV/AIDS patients by having CD4 > 350 cell/ul this therefore confirmed the immunosuppressive impact TB/HIV on immune system of the patients by reducing the CD4 cells in their system. However, people that are infected with HIV in developing countries develop TB as the symptom of AIDS. The two diseases represent a deadly combination by complimenting each other.

In Nigeria and other resource poor countries, it is solely being used as index of monitoring HIV progression though viral load is more reliable, save its huge cost in resource poor countries. In addition, the fact that CD4 values are influences by socio demographic, some personal biological and environmental factors could affect the prospect of its used in diagnoses and monitoring of HIV/TB co-infections. Latent TB infection has been known to revert to active TB following new infection in HIV infected people. TB has been shown to accelerate the progression of HIV disease.

'CD4 cell count is readily available unlike the viral load which better monitors but which many programmes could not afford. CD4 going up may be an index of an improving adherence to HAART based on calculated adherence percentage. Most PLWHAs in Nigeria would most likely be on first line drugs with good adherence strategies. Shifting to $2^{\text {nd }}$ line ART would have several implications for programmes and the health systems in terms of funding and monitoring to prevent and reduce the high mortality and morbidity known to be associated with TB-HIV co-infection compared to single case TB or HIV.

\section{Conclusion}

Co-infections of TB and HIV are common. The CD4 cell counts of patients attending TB clinic during the period October, 2009 to June, 2011 is affected by the co-infection of TB/HIV. Every HIV positive client should be considered having the potential of contracting TB. This underscores the importance of proper screening, monitoring and prevention of opportunistic infections most especially TB.

\section{Acknowledgement}

Author would like to thank the Medical Superintendent of Dr. Lawrence Henshaw Memorial Hospital, Calabar, all the Staff of Departmnet of Laboratory Services and all PLHIVs who gave consent towards participation in this research.

\section{References}

[1] Corbett EL, Watt CJ, Walker N. The growing burden of tuberculosis: global trends and interactions with the HIV epidemic. Arch Intern Med, 2003; 163: 1009-1021.

[2] World Health Organization WHO. Tuberculosis Fact sheet $\mathrm{N}^{\circ} 104$-Global and regional incidence. March 2006, Retrieved on 6 October 2006.

[3] World Health Organization. WHO Report Global TB control: Nigeria. In: WHO, editor. Geneva, Switzerland: WHO; 2008. Available from: http://www.who.int/globalatlas/predefinedReports/TB/PDF Fi les/nga.pdf [Last accessed on 23rd October 2008].

[4] Lawson L, Yassin MA, Thacher TD, Olatunji OO, Lawson JO, Akingbogun TI. Clinical presentation of adults with pulmonary tuberculosis with and without HIV infection in Nigeria. Scand J Infect Dis, 2007; 10: 1-6.

[5] Global Tuberculosis Control 2014" Available at www.who.int/tb/publications/global_report/_ Accessed November 12, 2015].

[6] "Implementing the WHO Stop TB Strategy: a handbook for national tuberculosis control programmes" Geneva, World Health Organization, 2008, - See more at: http://www.tbfacts.org/tb-hiv/\#sthash.DE69pWlm.dpuf.

[7] Luetkemeyer, A. "Tuberculosis and HIV", HIVInSite, http:/hivinsite.ucsf.edu/ - See more at: http://www.tbfacts.org/tb-hiv/\#sthash.DE69pWlm.dpuf.

[8] Suchindran, S. "Is HIV infection a Risk Factor for Multi-Drug Resistant Tuberculosis? A Systematic Review" PLoS one, May 2009, 4 (5): e5561 http://www.plosone.org/article/ - See more at: http://www.tbfacts.org/tbhiv/\#sthash.DE69pWlm.dpuf.

[9] Vazquez, G. J. C., D. E. Sada, M. E. Rivera, P. O. Narvaez and L. M. A. Salazar, 1994. Tuberculosis associated with HIV infection. Rev. Invest. Clin., 46: 473-477.

[10] Yassin, M. A., L. Takele, S. Gebresenbet, E. Girma, M. Lera, E. Lendebo and L. E. Cuevas, 2004. HIV and tuberculosis coinfection in the southern region of Ethiopia: A prospective epidemiological study. Scand J. Infect. Dis., 36: 670-673.

[11] Brown, M., G. Miiro and P. Nkurunziza, 2006. Schistosoma mansoni, nematode infections and progression to active tuberculosis among HIV-1-infected Ugandans. Am. J. Trop. Med. Hyg., 74: 819-825.

[12] World Health Organization, author. Global Tuberculosis Report 2014. Geneva, Switzerland: World Health Organization; 2014.

[13] World Health Organization, author. WHO Country Cooperation Strategy 2012-2015. Ethiopia: WHO Regional Office for Africa, Republic of Congo; 2013. http://www. afro.who.int/. 